\title{
Eat Healthy, Be Active Community Workshops implemented with rural Hispanic women
}

\author{
Janeth I. Sanchez ${ }^{1,2}$, Katherine J. Briant ${ }^{2}$, Samantha Wu-Georges³ ${ }^{3}$ Virginia Gonzalez ${ }^{3}$, Avigail Galvan³, \\ Sara Cole $2^{2^{*}}$ and Beti Thompson ${ }^{1,2}$
}

\begin{abstract}
Background: In the U.S., obesity disproportionately affects some racial/ethnic groups more than others; $42.5 \%$ of Hispanic adults are obese, compared to $32.6 \%$ of non-Hispanic whites (NHW). Research also shows that Mexican American women are $40 \%$ more likely to be overweight, as compared to NHW women. With high obesity rates among Hispanics, improving healthier lifestyle practices is an important step for reducing health disparities. The Eat Healthy, Be Active (EHBA) community workshops were developed to assist individuals in translating national nutrition and physical activity recommendations into action. Promotora-led EHBA workshops could be used to promote obesity-related health behavior lifestyle changes among Hispanics.

Methods: Hispanic women from rural communities in Washington state were recruited to participate in a six-week Promotora-led workshop series. This pilot study used a pre- and post-test study design to examine differences in healthy lifestyle knowledge and practices.

Results: A total of 49 Hispanic women participated in the workshops, of whom $45 \%$ were obese. Six-weeks after implementation of EHBA, women had improvements in healthy lifestyle practices, including an increase in nutrition label literacy, decrease in consumption of food eaten in restaurants, and an increase in the number of times a woman performed physical activity long enough to make them sweat.

Conclusion: The findings from this pilot study indicate that delivering EHBA workshops through promotoras is a feasible culturally relevant approach to promoting healthier lifestyle practices among Hispanic women. Further, focusing on females, who do the food shopping and preparation in their homes, may help increase awareness among whole families.
\end{abstract}

Keywords: Obesity, Nutrition, Physical activity, Hispanics, Promotoras

\section{Background}

In the US, over 15 million American adults are obese [1]. Data from the National Health and Nutrition Evaluation Survey (NHANES), indicate that obesity has been steadily increasing over the past few decades and by 2030 , obesity

\footnotetext{
${ }^{*}$ Correspondence: scole2@fredhutch.org

2 Fred Hutchinson Cancer Research Center, 1100 Fairview Ave. N., M3-B232, Seattle, WA 98166, USA

Full list of author information is available at the end of the article
}

rates are expected to rise by $33 \%[2,3]$. Especially alarming is the increasing significant positive linear trend in obesity rates among Hispanic women who demonstrate a $10 \%$ higher prevalence of obesity compared to nonHispanic white (NHW) women [4-6]. Obesity is a major public health concern for Hispanic women as it is a significant risk factor for the development of serious chronic conditions, such as coronary heart disease (CHD), diabetes, and cancer [7-9]. For example, although Hispanics have lower prevalence of CHD compared to NHWs, 
Hispanic women are likely to develop heart disease ten years earlier than NHW women and are less likely to be aware of their risk for CHD [10, 11]. Similarly, studies have consistently demonstrated an increasing prevalence of diabetes and cancer associated with increasing weight among Hispanic women [12-14]. Obesity increases the risk for developing breast, colorectal, and endometrium cancer among Hispanic women and has been linked to poorer breast cancer survival in this population $[15,16]$. Maintaining a healthy weight and engaging in regular physical activity are important approaches that can help reduce incidence and mortality from $\mathrm{CHD}$, diabetes, and cancer $[8,15]$.

Educating Hispanic women about obesity-related risk factors and the importance of adopting healthy behaviors is important since even a loss of $3 \%$ to $5 \%$ in weight can result in measurable improvement in managing chronic conditions and comorbidities [17, 18]. Increasing knowledge and awareness of risk factors and promotion of healthier lifestyles are key components of behavioral health interventions. Further, obesity-related health promotion efforts focusing on increasing consumption of healthier food options and increasing physical activity are of importance among sub-populations of Hispanic women who experience higher prevalence of diabetes, such as Mexican-American women [6].

Unfortunately, Hispanic women, especially those residing in rural geographical areas, continue to face significant barriers that may limit the ability to achieve desired health outcomes. For example, rural Hispanic communities face several healthcare challenges, including shortages in healthcare-related services and personnel, as well as a lack of access to culturally appropriate resources and information, that aggravate obesity incidence and contribute to poor obesity-related outcomes [19]. The lack of community resources in rural areas (e.g., poor infrastructure to encourage physical activity, lack of access to financially accessible healthy foods at local supermarkets) further exacerbate poor health outcomes among Hispanic women [19-21]. Identifying and implementing culturally tailored health promotion interventions may assist in improving obesity-related outcomes among Hispanic women living in rural areas.

To address such barriers, trends in the field of health promotion have shifted from clinically delivered individual interventions to efforts that use a community-based approach that encompasses social and environmental factors to deliver interventions in community settings [22-24]. Research suggests that community-based interventions promote healthy lifestyle behaviors among Hispanic women [23, 25]; for example, weight management programs delivered in community settings have proven to be effective in promoting weight loss, improving obesity-related risk behaviors, and decreasing BMI among Hispanic women immigrants [26].

Moreover, promotora-delivered interventions promote healthier dietary habits and increased performance of physical activity among Hispanic women [26-28]. The WISEWOMAN program, for example, utilized a promotora-led approach to recruit, educate and deliver a lifestyle health promotion program among Hispanic women living in California. Women receiving the intervention demonstrated improved eating habits and physical activity, as well as a reduced 10-year CHD risk [29]. Another example, the ENLACE program, was a multisite research study aimed to deliver a community-based physical activity intervention to Hispanic women using a promotoraled delivery model [30]. Hispanic women who received the ENLACE intervention increased their physical activity by $56.4 \%$ compared to $48.8 \%$ among the women in the control group [30].

Much of the success of promotora-led behavioral interventions among Hispanic women is linked to the promotoras' ability to be culturally sensitive and to lead and motivate participants with culturally relevant tools, knowledge, and support [28]. Promotoras appear to serve as cultural mediators and role models who provide emotional and social support in ways that make Hispanic women feel secure and comfortable, thus facilitating acquisition of the skills that enable healthy lifestyle changes [28, 31]. Overall, findings from systematic reviews of promotora-led interventions provide evidence of their effectiveness in improving health-related behaviors and outcomes [32-34].

Given the burden of obesity-related outcomes faced by Hispanic women residing in rural areas, there is a continued need for community-based promotora-led health promotion interventions that are culturally adapted and tailored for rural populations. One such communitybased program, the Eat Healthy, Be Active Community Workshops (EHBA) was developed by the U.S. Department of Health and Human Services' Office of Disease Prevention and Health Promotion. The focus of EHBA is to educate and assist individuals from varying literacy levels and socioeconomic backgrounds in gaining skills to adopt and sustain recommended nutrition and physical activity behaviors to promote good health and reduce risk of chronic diseases [35]. The curriculum has been tested among diverse populations in the United States; however, no study to date has evaluated its effectiveness among rural Hispanics. The aim of the pilot study was to assess the feasibility of implementing a promotora-led EHBA intervention to improve obesity-related behaviors among Hispanic women residing in a predominantly Hispanic rural community. 


\section{Methods}

\section{Setting}

The Lower Yakima Valley of Washington State (the Valley) is a region known for its vast agricultural industry, rurality, and predominant Hispanic population; approximately $68 \%$ of the population in the Valley is Hispanic and the majority are of Mexican descent [36]. Hispanics living in the Valley have lower socioeconomic status, lower educational attainment and limited access to health care services that contribute to poor health outcomes [37].

\section{Recruitment and participation}

The target for this pilot intervention was 50 Hispanic women [38], 18 years or older, who were responsible for the food shopping and food preparation in their homes. To recruit individuals, flyers were placed in various locations throughout the Valley, including at grocery and convenience stores, and community centers. Individuals were also recruited at local community events, including health fairs in the region. Once recruited, participants were registered to participate in one of the workshop series held in 2015. Of the 50 women who agreed to participate, two women did not complete the followup questionnaire. In addition, the number of women who responded to each item varied from baseline to follow-up.

\section{Identifying an intervention program}

Researchers in the Valley use community-based participatory research (CBPR) methods to address health disparities experienced by the Hispanic population. A Community Advisory Board (CAB) in the Valley noted the high prevalence of obesity in the region and recommended that a research effort be initiated to address obesity rates in the community. The $\mathrm{CAB}$ recommended that a promotora approach be part of the intervention. After a search for validated programs, we identified the Eat Healthy, Be Active Community Workshops (EHBA) program as being appropriate for the intervention [35].

\section{EHBA intervention}

The Eat Healthy, Be Active Community Workshops (EHBA) is an educational curriculum developed in English and Spanish to promote consumption of healthy balanced diets and increased physical activity [35]. The curriculum was designed using low-literacy principles and pilot tested at ten different sites across the US, including state cooperative extensions, faith-based organizations, worksite wellness programs, and parent groups. EHBA workshops consist of six one-hour interactive workshops and include the following sections: 1) Eating Healthy Food That Tastes Great, 2) Quick, Healthy
Meals and Snacks, 3) Eating Healthy on a Budget, 4) Tips for Losing Weight and Keeping it Off, 5) Making Healthy Eating Part of Your total Lifestyle, and 6) Physical Activity is Key to Living Well. The EHBA curriculum includes a lesson plan, learning objectives for each workshop, talking points, hand-on-activities, videos and handouts. The workshops may be taught by various trained professionals, including promotores, and may take place in a variety of community settings.

\section{Training}

The Fred Hutchinson Cancer Research Center's (Fred Hutch) Center for Community Health Promotion (CCHP) has a Community Health Educator (CHE) whose role is to train local community health workers (called promotores) to implement health promotion interventions among local Hispanic groups or individuals in the Valley. The cadre of promotores are trusted individuals from the Valley who are bilingual and bicultural. The promotores are trained in community-based participatory research $(\mathrm{CBPR})$, collecting survey data, and facilitating educational interventions and community outreach. The CHE trained six promotores to implement the EHBA. Each promotor/a received a two-day training in which they went over all six workshops, engaged in role-playing to answer questions, discussed various features of the program, and obtained certification in the content of the curriculum. For this pilot, EHBA was facilitated and implemented by two female protomoras of those trained.

\section{Implementation}

We offered the EHBA workshop series at five different times between January 2015 and December 2015. Participants in the workshops were consented and were asked to complete a pre-test (baseline) at the beginning of Workshop One. Each session began with an Icebreaker activity and an introduction explaining the purpose of the workshop and review of the learning objectives. Promotores reviewed specific workshop-related talking points, handouts, presented a video and facilitated activities (i.e., MyPlate drawing, how much sugar in a soda?, Using a slow cooker) during each session. Participants were asked to complete a post-test (follow-up) at the end of the six-week series.

\section{Study design and measures}

For this project, a pre- and post-test study design was used to assess the feasibility of implementing EHBA among Hispanic women and its ability to change obesityrelated behaviors from baseline to follow-up. Items for the questionnaires were taken from a previous diabetes study [39]. Baseline questionnaire (pre-test) included items on demographics, such as age, education, race, 
language of preference, country of birth, length of residency in the Valley, regular source of health care, and access to health care. Both questionnaires contained health behavior items, including two items on nutrition label literacy; an item assessing knowledge about interpreting serving sizes and knowledge of calorie intake based on serving size. Consumption of sugar-sweetened drinks was ascertained via four items, with two items, one for soda and another for non-soda, assessing how many ounces were consumed each day during the past 30 days and similarly two items assessing how often these drinks were consumed (e.g., a day, a week, a month). Two composite variables were then created to assess consumption of soda and non-soda sugary drinks per week. Fruit and vegetable (F\&V) consumption was ascertained at baseline and follow-up using six items from the Thompson and Subar instrument [40]; three items for fruit consumption and three for vegetable consumption. Items asked how often the woman ate F\&Vs (other than potatoes) during the past 30 days, the frequency (day, week, month) of consumption, and how many cups of F\&Vs were consumed. Although the Thompson and Subar instrument used actual serving size, this was not easy for our participants to understand. Thus, we used 'cups' instead of serving size as this was familiar to participants. One composite variable for F\&Vs was developed to measure the number of cups of F\&Vs consumed per day for consistency across participants. Participants also were asked about food security; that is, how often they felt worried or stressed about having enough money to buy nutritious meals during the past 12 months. Finally, the frequency with which women ate food prepared at restaurants was assessed via two items; one item assessed how often, on average, the participant ate foods that were prepared in restaurants (no/yes; yes variable included 'rarely') and the second item assessed the number of times per week eaten food prepared in a restaurant among those who responded yes to prior question.

We captured amount of physical activity performed during leisure time using four items. Three items assessed the amount of physical activity performed by level of intensity (e.g., strenuous, moderate, or mild): 'how many times per week do you perform physical activity for more than ten minutes during free time outside of work'. Another item assessed how often the participant engaged in regular physical activity long enough to sweat during the last seven days using a four-point Likert scale (never, rarely, sometimes, often).

Height and weight for participants at both time points used a SECA medical scale with stadiometer. CDC Standard weight status categories, developed by the Centers for Disease Control and Prevention, associated with BMI ranges for adults were used to calculate participant BMI categories (Normal: 18.5-24.9; Overweight: 25.0-29.9; Obese: 30.0+) [41]. We also assessed perceived selfweight at baseline and follow-up using one item asking about how the participant felt about her weight (overweight, slightly overweight, just about the right weight for me and slightly underweight).

\section{Data collection}

Responses to all the questionnaires were collected and managed using REDCap electronic data capture tool. REDCap is a secure online software application designed to support and facilitate data capture for research studies [42]. The Fred Hutchinson Cancer Research Center Institutional Review Board approved this study (File \#7293).

\section{Analytic plan}

Quantitative data analyses were performed using STATA 14.2 (STATACorp, 2014). Study analysis focused on assessing workshop participation and comparing differences in obesity-related behaviors from baseline to follow-up. Descriptive statistics are presented using proportions of participants who completed the baseline and follow-up questionnaires. McNemar's exact test for matched-paired binary data was performed to assess differences in marginal frequencies for nutrition label literacy, consumption of sugary drinks, and meeting national recommendations for F\&V consumption. Paired-sample $t$ test was performed to assess differences in mean consumption of F\&Vs, performance of physical activity, and eating out at restaurants from baseline to follow-up. Differences in mean weight change and BMI from baseline and follow-up were also assessed.

\section{Results \\ Participant characteristics Demographics}

A total of 50 women were recruited to participate in the pilot EHBA workshops, however, one woman self-identified as non-Hispanic White and was excluded from all analyses. All women included in this study $(n=49)$, selfidentified as Hispanic; ages ranged from 19 to 75 years old (Table 1). Most participants (67.4\%) reported having an education level 'less than high school degree'. Among the women, $82.0 \%(n=41)$ reported Spanish as their preferred language to speak and read and $82 \%$ completed the surveys in Spanish. One participant reported they preferred both English and Spanish. The majority of the participants were born in Mexico (70.0\%) and reported living in the Valley for more than five years (79.6\%). Over $40 \%(n=21)$ had no health insurance of any kind. 
Table 1 Participant demographics $(\mathrm{N}=49)$

\begin{tabular}{|c|c|c|}
\hline & Total & $\%$ \\
\hline \multicolumn{3}{|l|}{ Age } \\
\hline $18-34$ & 15 & 30.6 \\
\hline $35-44$ & 19 & 38.8 \\
\hline $45-64$ & 14 & 28.6 \\
\hline $65+$ & 1 & 2.0 \\
\hline \multicolumn{3}{|l|}{ Education } \\
\hline$<\mathrm{HS}$ degree & 33 & 67.4 \\
\hline HS degree & 8 & 16.3 \\
\hline >HS degree & 8 & 16.3 \\
\hline \multicolumn{3}{|l|}{ Preferred language } \\
\hline Spanish & 41 & 82.0 \\
\hline English & 9 & 18.0 \\
\hline \multicolumn{3}{|l|}{ Country of origin } \\
\hline U.S & 12 & 24.0 \\
\hline Mexico & 35 & 70.0 \\
\hline Other & 3 & 6.0 \\
\hline \multicolumn{3}{|c|}{ Length of time living in U.S } \\
\hline$<1 \mathrm{yr}$ & 6 & 12.2 \\
\hline 1 to 5 years & 4 & 8.2 \\
\hline$>5$ years & 39 & 79.6 \\
\hline \multicolumn{3}{|l|}{ Health Insurance } \\
\hline Private & 10 & 20.4 \\
\hline Medicare & 4 & 8.2 \\
\hline Medicaid & 13 & 26.5 \\
\hline Other & 1 & 2.0 \\
\hline No Health Insurance & 21 & 42.9 \\
\hline
\end{tabular}

\section{Health knowledge and behaviors Nutrition health literacy}

There was an increase in the number of women who demonstrated nutrition label literacy from baseline to follow-up. Specifically, there were 38 women at follow-up who interpreted calorie content per serving size in a container from the nutrition label correctly compared to 25 women at-time of baseline ( $77.6 \%$ vs $51.0 \%$, respectively); further, the proportion of women who correctly identified daily calorie intake based on serving size increased from baseline to follow-up (76.1\% vs 51.0\%) (Table 2).

\section{Food insecurity}

Of the women who responded to this question, 69.4\% stated experiencing stress about having enough money to purchase nutritious foods at baseline and followup. Among the women who responded at both baseline and follow-up $(n=47), 74.5 \%$ had no change in their food insecurity status, $14.9 \%$ reported food insecurity at follow-up but not at baseline, and $10.6 \%$ reported food insecurity at baseline, but not after completing the EHBA workshop.

\section{Consumption of sugar sweetened drinks}

For soda and sugary drinks, $35.6 \%(n=16)$ of the women reported a decrease in the number of ounces consumed per week from baseline to follow-up; however, $44.4 \%$ $(\mathrm{n}=20)$ had no change and $20.0 \%(\mathrm{n}=9)$ reported an increase (Table 3). For non-soda sugary drinks, 37.8\% $(n=17)$ of the women reported a decrease in the number of ounces consumed per week, 33.3\% $(n=15)$ indicated having no change and $28.9 \%$ reported an increase. The average number of ounces consumed per week from baseline to follow-up decreased for both soda and nonsoda sugary drinks (Table 4).

\section{Fruit and Vegetable (F\&V) consumption}

Among our participants, $61.7 \%$ of the women increased the number of F\&V cups consumed per day from baseline to follow-up, while $36.2 \%$ and $2.1 \%$ reported a decrease and no change, respectively, in the number of F\&V cups consumed per day (Table 3 ). The mean number of cups of F\&Vs consumed per day increased by 0.29 cups from baseline to follow-up (Table 4). A total of 53\% of the women consumed the minimum F\&V cups per day (i.e., 2.5) recommended for having a reduced risk of coronary heart.

\section{Eating out at restaurants}

From baseline to follow-up, the proportion of women who reported eating food prepared in restaurants did not change (Table 3 ). In addition, there was no difference in the mean number of women who reported eating food prepared in restaurants from baseline to follow-up (Table 4).

Table 2 Changes in the proportion of women with nutrition label literacy and food insecurity from baseline to follow-up

\begin{tabular}{|c|c|c|c|c|c|}
\hline & $\begin{array}{l}\text { Pre } \\
\text { n (\%) }\end{array}$ & $\begin{array}{l}\text { Total \# of respondents } \\
\mathrm{N}\end{array}$ & $\begin{array}{l}\text { Post } \\
\text { n (\%) }\end{array}$ & $\begin{array}{l}\text { Total \# of respondents } \\
\mathrm{N}\end{array}$ & $p$ value \\
\hline \multicolumn{6}{|l|}{ Nutrition label literacy } \\
\hline Correctly identified calorie content & $25(51.0)$ & 49 & $38(77.6)$ & 49 & .002 \\
\hline Correctly identified daily calorie intake & $25(51.0)$ & 46 & $35(76.1)$ & 46 & .043 \\
\hline Experienced food security & $34(69.4)$ & 49 & $34(69.4)$ & 47 & .569 \\
\hline
\end{tabular}


Table 3 Proportion of Hispanic women with changes in health behaviors and health status from baseline to post-EHBA Workshop implementation

\begin{tabular}{llll}
\hline & $\begin{array}{l}\text { Decrease } \\
\mathbf{n}(\%)\end{array}$ & $\begin{array}{l}\text { Increase } \\
\mathbf{n}(\%)\end{array}$ & $\begin{array}{l}\text { No change } \\
\mathbf{n}(\%)\end{array}$ \\
\hline $\begin{array}{l}\text { Nutrition } \\
\text { Sugary drink consumption (soda) }\end{array}$ & $16(35.6 \%)$ & $9(20.0 \%)$ & $20(44.4 \%)$ \\
Sugary drink consumption (non-soda) & $17(37.8 \%)$ & $29(61.7 \%)$ & $1(2.13 \%)$ \\
F\&V consumption & $17(36.2 \%)$ & $17(36.2 \%)$ & $17(36.2 \%)$ \\
Eating food prepared in restaurants & 0 & 0 & $49(100 \%)$ \\
Performed Physical Activity (PA) & & $13(27.7 \%)$ & $26(55.3 \%)$ \\
$\quad$ Leisure Strenuous & $8(17.0 \%)$ & $17(36.2 \%)$ & $14(29.8 \%)$ \\
Leisure Moderate & $16(34.0 \%)$ & $20(42.6 \%)$ & $12(25.5 \%)$ \\
Leisure Mild & $15(31.9 \%)$ & $26(55.3 \%)$ & $14(29.8 \%)$ \\
Engaged in regular PA enough to sweat & $7(14.9 \%)$ & $18(39.1 \%)$ & $3(6.5 \%)$ \\
Weight \& BMl & & $19(41.3 \%)$ & $5(10.9 \%)$ \\
Weight (measured in pounds) & $25(54.3 \%)$ & Worse status & No change \\
BMl (kg/m $\left.{ }^{2}\right)$ & $22(54.3 \%)$ & $6(12.8 \%)$ & $33(70.2 \%)$ \\
\hline
\end{tabular}

Table 4 Mean changes in health behaviors and health status from baseline to follow-up

\begin{tabular}{|c|c|c|c|c|c|c|c|}
\hline & \multicolumn{3}{|c|}{ Pre } & \multicolumn{3}{|c|}{ Post } & \multirow[t]{2}{*}{$p$ value } \\
\hline & $\mathbf{n}$ & Mean & $(95 \% \mathrm{Cl})$ & $\mathbf{n}$ & Mean & $(95 \% \mathrm{Cl})$ & \\
\hline \multicolumn{8}{|l|}{ Nutrition } \\
\hline Sugary Drinks (soda) ${ }^{a}$ & 49 & 1.53 & $(0.97,2.10)$ & 45 & 1.33 & $(0.82,1.85)$ & 0.517 \\
\hline Sugary Drinks (non-soda) ${ }^{a}$ & 49 & 1.96 & $(1.36,2.56)$ & 45 & 1.80 & $(1.21,2.40)$ & 0.682 \\
\hline F\&V consumption ${ }^{b}$ & 48 & 3.67 & $(17.15,34.22)$ & 46 & 3.99 & $(20.33,35.50)$ & 0.293 \\
\hline Ate food from restaurants ${ }^{c}$ & 49 & 0.90 & $(0.81,0.99)$ & 48 & 0.90 & $(0.81,0.99)$ & - \\
\hline \multicolumn{8}{|l|}{ Physical activity } \\
\hline Leisure Strenuous $^{d}$ & 47 & 0.68 & $(0.27,1.10)$ & 47 & 1.10 & $(0.55,1.66)$ & 0.182 \\
\hline Leisure Moderate $^{d}$ & 47 & 1.94 & $(1.24,2.63)$ & 47 & 2.02 & $(1.45,2.60)$ & 0.852 \\
\hline Leisure Mild $^{d}$ & 47 & 2.26 & $(1.47,3.04)$ & 47 & 3.06 & $(2.26,3.87)$ & 0.112 \\
\hline Engage in regular PA enough to sweat ${ }^{\mathrm{e}}$ & 47 & 1.13 & $(0.84,1.42)$ & 47 & 1.83 & $(1.52,2.14)$ & $<.001$ \\
\hline $\operatorname{BMI}\left(\mathrm{kg} / \mathrm{m}^{2}\right)$ & 48 & 30.2 & $(28.34,31.98)$ & 46 & 30.1 & $(28.30,31.97)$ & .809 \\
\hline
\end{tabular}

a Ounces consumed per week

b Number of cups of F\&V consumed per day

c Number of women who ate food prepared in restaurants

d Times per week physical activity was performed

e Frequency of physical activity performed per week (never $=0$, rarely $=1$, sometimes $=2$, often $=3$ )

- No difference in mean from baseline to follow-up

\section{Physical activity}

Changes in the number of times per week a woman reported performing physical activity from baseline to follow-up varied by intensity. At follow-up, 27.7\% $(\mathrm{n}=13)$ of women reported an increase in the number of times per week they performed strenuous leisure physical activity, 36.2\% $(\mathrm{n}=17)$ increased moderate leisure physical activity, and $42.6 \%(n=20)$ increased their mild physical activity performed per week (Table 3 ). Among the participants, there were small increases in the mean frequency of physical activity performed per week from baseline to follow-up (vigorous: 0.68 to 1.10 ), moderate: 
1.94 vs 2.02 , and mild: 2.26 vs 3.06 ) (Table 4 ). There was also a significant increase from 'rarely' to 'sometimes' in the mean frequency women performed regular physical activity long enough to make them sweat in the last 7 days during free time $($ mean $=1.13,95 \% \mathrm{CI}: 0.84-1.42$ to mean $=1.83)$, $95 \%$ CI: $1.52-2.14$ ).

\section{Weight and obesity status}

We assessed weight at baseline $(\mathrm{n}=49$, mean $=167.5)$ and follow-up $(\mathrm{n}=46$, mean $=168.7)$, as well as changes in BMI for 46 of the women for whom we were able to capture this data at both time points. More than half of the women with complete data $(54.3 \%)$ had a decrease in weight ranging from 0.2 to $11.4 \mathrm{lb}$, while $39.1 \%$ had an increase in weight (Table 3). There were no meaningful changes in mean weight and BMI from baseline to follow-up (Table 4). With regard to changes in perceived self-weight from baseline to follow-up, $70.2 \%$ reported no change, $17.0 \%$ reported a better self-perceived status, while $12.8 \%$ reported a worse status (Table 3 ).

\section{Discussion}

This pilot study assessed the feasibility of implementing the promotora-led Eat Healthy, Be Active (EHBA) community workshops to improve nutrition label literacy and assist in the adoption of healthy behaviors among Hispanic women residing in a rural geographical area. The proportion of Hispanic women who demonstrated an ability to read nutrition labels accurately increased from $51 \%$ at baseline to more than $75 \%$ at follow-up, which is a positive predictor of dietary quality [43]. Among Hispanic populations, less than $20 \%$ demonstrate adequate comprehension of food labels [44]. Improving nutrition label literacy is an important step for addressing high obesity rates among Hispanic women since studies have found that individuals who check food labels when purchasing food are more likely to report having healthy dietary practices compared to non-label users [45]. For many Hispanic populations, low levels of numeracy and literacy may further limit their ability to comprehend complex nutrition labels [46]. Although, the EHBA workshops do not address health literacy or other factors associated with low educational attainment, the workshops provide an avenue for improving a Hispanic woman's ability to understand useful information contained within food nutrition labels and to translate that into the management of obesity-related outcomes.

Sugar-sweetened beverages are major sources of added sugar in the diets of Americans, accounting for $36 \%$ of added sugar intake [47]. A higher consumption of sugar-sweetened beverages is associated with weight gain, risk of type 2 diabetes and hypertension $[47,48]$. In our study, 16 women $(35.6 \%)$ decreased their consumption of sugar-sweetened soda drinks over a six-week period, while 9 (20.0\%) had an increase in the number of ounces of soda consumed per week from baseline to follow-up. However, among the women who had an increase, all women consumed less than 24 ounces of soda per week. Similar results were observed for non-soda sugary drinks. While the proportion of women who decreased their consumption of sugar-sweetened drinks may seem small, EHBA could assist Hispanic women in promoting a reduction in the consumption of such drinks overall. This is particularly important given that a reduction in consumption of one serving of sugary drinks per day has been shown to reduce blood pressure [48].

National organizations recommend making half of the plate F\&Vs and choosing a variety of red, orange and dark-green vegetables for meals. Increasing the consumption of healthy foods is a main goal of the EHBA curriculum. Consumption of F\&V cups per day increased among 29 women (61.7\%). Although 17 women reported a decrease of $F \& V$ consumption per day, $35.3 \%$ of these consumed at least 2.5 cups of F\&V per day. Evidence suggests that intake of at least 2.5 cups of F\&Vs per day is associated with a reduced risk of coronary heart disease, including heart attacks and stroke [49]. Among the women in this study, the mean F\&V consumption per day at baseline was approximately one cup higher than the $\mathrm{F} \& \mathrm{~V}$ intake recommendations to reduce $\mathrm{CHD}$.

The Physical Activity Guidelines for Americans recommends at least $75 \mathrm{~min}$ of vigorous intensity activity or $150 \mathrm{~min}$ of moderate intensity activity per week for adults [35]. The first five EHBA workshops include a one to two minute talking point about physical activity with weekly tips on how to incorporate physical activity into their daily routines. Workshop Six focuses entirely on educating individuals about the benefits of physical activity, provides recommendations for identifying and performing aerobic and strengthening activities, as well as assist participants in developing and sustaining a successful plan for performing daily physical activity. More than $25 \%$ of the women increased the number of times per week they performed leisure strenuous, moderate, and mild physical activity. After participating in the workshop, the mean number of times per week women performed strenuous and mild physical activity increased. There was a significant increase in the frequency a woman performed physical activity enough to make them sweat from rarely to sometimes. Therefore, EHBA could assist Hispanic women in identifying ways to increase their physical activity at home, even in areas with limited access to parks and community centers. Previous qualitative studies have identified lack of time, being tired and lack of self-discipline as barriers reported 
by Hispanic adults for not performing physical activity [50].

Similar to national obesity rates among Hispanics, $45 \%$ of the women participating in this pilot study were obese. Obesity was a secondary outcome of this study and, given the short study period of six weeks, we did not expect significant changes in weight or BMI from baseline to follow-up. However, there was a decrease in weight measured among $54.3 \%$ of the women, which also translated to a decrease in BMI. Among older women (e.g., 50 years of age and older), behavioral changes related to diet and exercise improves body weight and adiposity, especially if such lifestyle changes are maintained for over a year [51]. Other studies, such as the Diabetes Prevention Program (DPP) also provide strong evidence of the effectiveness of diet and exercise lifestyle interventions on decreasing incidence of obesity and chronic conditions over three years [51]. Thus, future implementation of EHBA curriculum should include additional follow-ups (e.g., 6-month and 12-month follow-ups) to assess sustained healthy behaviors and decreases in weight and BMI to better understand the long-term impact of EHBA participation on reducing BMI.

Inequities in access to healthy choices where Hispanics live and work contribute to the high rates of obesity among these populations [52]. Food insecurity was prevalent in this study, with $69.4 \%$ of the participants stating they felt worried or stressed about having enough money to buy nutritious meals. Future implementation of EHBA should be coupled with efforts that address social, environmental and financial barriers that limit access to healthy foods and places to perform physical activity. Strategies to address disparities in obesity rates among Hispanics must include sustained and community collaborative approaches that address the challenges that stem from social risk factors, which influence access to healthy and affordable food, as well as promote physical activity [52].

Limitations. This pilot study has several limitations. A total of 49 women participated in this study, which is a small number but consistent with the samples size for pilot studies [38]. There was variation in the number of women who responded to specific items in each questionnaire. Variation in the responses may be associated with factors unrelated to the actual content of the workshops, such as personal and cultural preferences to respond to specific items. In addition, information on nutrition and physical activity are based on self-reported data, which are susceptible to several types of biases (e.g., recall bias, measurement bias, and social desirability bias). For example, food frequency intake and amount of physical activity performed may be biased by a participant's ability to recall actual behaviors in the last 30 days [53]. Participants may also respond to items in a manner that they may deem to be socially desirable, such as underreporting consumption of sugary sweetened drinks and/or overreporting intake of $F \& V s$ and amount of physical activity performed [54]. Nonetheless, using self-reported questionnaire items to capture food intake and physical activity is a common method due to its reliability and validity in assessing behaviors (e.g., food consumption), low burden on participants, and ease of administering survey [54]. Although we did not have a control or comparison group, this is common in pilot studies. Future implementation of EHBA in rural Hispanic populations should include a larger sample size of women with comparison or control groups to assess the impact of EHBA. Additionally, the final assessment of the pilot study was at the end of the six weeks of workshops, which is a short time to achieve large improvements in health behaviors; it is important for a longer-term assessment of whether participants developed or maintained positive behavior changes over time.

\section{Conclusion}

A promotora-led Eat Healthy Be Active community workshops can be feasibly implemented among Hispanic women residing in rural geographical areas to improve nutrition label literacy, as well as to promote behavioral changes that may be linked to obesity reduction. The average change in obesity-related behaviors from baseline to follow-up were in the appropriate direction. Larger studies with longer follow-up are needed to fully understand the impact of this curriculum on members of the Hispanic population in rural settings.

\section{Abbreviations}

NHW: Non-Hispanic white; EHBA: Eat Healthy Be Active; CHD: Coronary heart disease; CBPR: Community based participatory research; CAB: Community advisory board; CHE: Community health educator; F\&V: Fruits and vegetables.

\section{Acknowledgements}

We would like to thank all the women who agreed to participate in this pilot study. We would also like to thank the promotoras who led the EHBA workshops. Finally, none of this could have been done without the strong participation of the Community Advisory Board, which supported this intervention and its implementation.

\section{Authors' contributions}

VG and AG collected data, assisted in designing the intervention of the study, and revised drafts of the work. SWG completed initial data analysis and contributed to drafts of the paper. JS, BT, and KB all designed the original study, conducted formal analysis, and headed the manuscript writing. SC revised the final draft, contributed to the analysis, and assisted in the interpretation of the work. All authors read and approved the final manuscript and agreed to be accountable for the work.

\section{Funding}

This project was supported by grant numbers U54 CA153502, P30 CA01570437S5, and T32 CA092408 from the National Cancer Institute (NCI) and the Institute of Translational Health Science grant (UL1 RR025014) from the National Center for Research Resources (NCRR) at the National Institutes of Health (NIH). 
The content is solely the responsibility of the authors and does not necessarily represent the official views of the NCI, NCRR or the NIH.

\section{Availability of data and materials}

The datasets used and/or analyzed during the current study may be available from the corresponding author on reasonable request.

\section{Ethics approval and consent to participate}

This intervention was reviewed by the Institutional Review Board at Fred Hutchinson Cancer Research Center (IR File \#7293). Written informed consent was obtained from all participants in their preferred language (English or Spanish).

\section{Consent for publication}

Not applicable.

\section{Competing interests}

The authors declare no potential conflicts of interest.

\section{Author details}

${ }^{1}$ Department of Health Services, University of Washington School of Public Health, Box 357230, Seattle, WA 98195, USA. ${ }^{2}$ Fred Hutchinson Cancer Research Center, 1100 Fairview Ave. N., M3-B232, Seattle, WA 98166, USA.

${ }^{3}$ Fred Hutchinson Cancer Research Center - Center for Community Health Promotion, 320 N. 16th Street, Sunnyside, WA 98944, USA.

Received: 3 December 2019 Accepted: 20 December 2020 Published online: 13 January 2021

\section{References}

1. Ogden CL, Carroll MD, Fryar CD, Flegal KM. Prevalence of obesity among adults and youth: United States, 2011-2014. 2015.

2. National Center for Health Statistics. National Health and Nutrition Examination Survey Fact Sheet. 2016.

3. Flegal KM, Kruszon-Moran D, Carroll MD, Fryar CD, Ogden CL. Trends in obesity among adults in the United States, 2005 to 2014. JAMA. 2016;315(21):2284-91.

4. National Hispanic Caucus of State Legislator. Hispanic Obesity: An American Crisis. Hispanic Obesity Initiative. 2010

5. Hales CM, Fryar CD, Carroll MD, Freedman DS, Aoki Y, Ogden CL. Differences in obesity prevalence by demographic characteristics and urbanization level among adults in the United States, 2013-2016. JAMA. 2018;319(23):2419-29.

6. Guendelman S, Weintraub MR, Kaufer-Horwitz M. Weight loss success among overweight and obese women of mexican-origin living in Mexico and the United States: a comparison of two national surveys. J Immigr Minor Health. 2017;19(1):41-9.

7. National Heart $\mathrm{L}$, and Blood Institute. What are overweight and obesity? 2012.

8. Wolin KY, Carson K, Colditz GA. Obesity and cancer. Oncologist. 2010;15(6):556.

9. Nguyen NT, Nguyen X-MT, Lane J, Wang P. Relationship between obesity and diabetes in a US adult population: findings from the National Health and Nutrition Examination Survey, 1999-2006. Obesity Surg. 2011:21(3):351-5

10. American Heart Association. Heart Disease Statistics at a Glance. 2017.

11. Balfour PC Jr, Ruiz JM, Talavera GA, Allison MA, Rodriguez CJ. Cardiovascular disease in Hispanics/Latinos in the United States. J Latina/o Psychol. 2016:4(2):98.

12. Crawford MA, Mendoza-Vasconez AS, Larsen BA. Type II diabetes disparities in diverse women: the potential roles of body composition, diet and physical activity. Women's Health. 2015;11(6):913-27.

13. Connor AE, Baumgartner RN, Pinkston C, Baumgartner KB. Obesity and risk of breast cancer mortality in Hispanic and Non-Hispanic white women: the New Mexico Women's Health Study. J Women's Health. 2013;22(4):368-77.

14. American Diabetes Association. Diabetes among Hispanics: All are not equal. 2014.
15. Wenten M, Gilliland FD, Baumgartner K, Samet JM. Associations of weight, weight change, and body mass with breast cancer risk in Hispanic and non-Hispanic white women. Ann Epidemiol. 2002;12(6):435-44.

16. National Cancer Institute. Obesity and Cancer Risk. 2012.

17. Members EP, Jensen MD, Ryan DH, et al. Executive summary: guidelines (2013) for the management of overweight and obesity in adults: a report of the American College of Cardiology/American Heart Association Task Force on Practice Guidelines and the Obesity Society published by the Obesity Society and American College of Cardiology/American Heart Association Task Force on Practice Guidelines. Based on a systematic review from the The Obesity Expert Panel, 2013. Obesity. 2014;22(S2):S5-S39.

18. Ryan DH, Yockey SR. Weight loss and improvement in comorbidity: differences at 5\%,10\%, 15\%, and over. Curr Obesity Rep. 2017;6(2):187-94.

19. Seguin R, Connor L, Nelson M, LaCroix A, Eldridge G. Understanding barriers and facilitators to healthy eating and active living in rural communities. J Nutr Metab. 2014;2014.

20. Trivedi T, Liu J, Probst JC, Merchant A, Jones S, Martin AB. Obesity and obesity-related behaviors among rural and urban adults in the USA. 2015.

21. Hub RHI. Rural obesity and weight control. 2018.

22. Ockene JK, Edgerton EA, Teutsch SM, et al. Integrating evidence-based clinical and community strategies to improve health. Am J Prev Med. 2007;32(3):244-52.

23. Koniak-Griffin D, Brecht M-L, Takayanagi S, Villegas J, Melendrez M, Balcázar H. A community health worker-led lifestyle behavior intervention for Latina (Hispanic) women: feasibility and outcomes of a randomized controlled trial. Int J Nurs Stud. 2015;52(1):75-87.

24. Baker PR, Francis DP, Soares J, Weightman AL, Foster C. Community wide interventions for increasing physical activity. Cochrane Database Syst Revi. 2015(1)

25. Merzel C, D'Afflitti J. Reconsidering community-based health promotion: promise, performance, and potential. Am J Public Health. 2003;93(4):557-74.

26. Cherrington AL, Willig AL, Agne AA, Fowler MC, Dutton GR, Scarinci IC. Development of a theory-based, peer support intervention to promote weight loss among Latina immigrants. BMC Obesity. 2015;2(1):17.

27. Mier N, Ory MG, Medina AA. Anatomy of culturally sensitive interventions promoting nutrition and exercise in Hispanics: A critical examination of existing literature. Health Promot Pract. 2010;11(4):541-54.

28. Albarran CR, Heilemann MV, Koniak-Griffin D. Promotoras as facilitators of change: Latinas' perspectives after participating in a lifestyle behaviour intervention program. J Adv Nurs. 2014:70(10):2303-13.

29. Hayashi T, Farrell MA, Chaput LA, Rocha DA, Hernandez M. Lifestyle intervention, behavioral changes, and improvement in cardiovascular risk profiles in the California WISEWOMAN project. J Women's Health. 2010;19(6):1129-38.

30. Parra-Medina D, Messias DKH. Promotion of physical activity among Mexican-origin women in Texas and South Carolina: an examination of social, cultural, economic, and environmental factors. Quest. 2011;63(1):100-17.

31. Reinschmidt KM, Hunter JB, Fernandez ML, Lacy-Martínez CR, Guernsey de Zapien J, Meister J. Understanding the success of promotoras in increasing chronic diseases screening. J Health Care Poor Underserv. 2006;17(2):256-64

32. Gibbons MC, Tyus NC. Systematic review of US-based randomized controlled trials using community health workers. Prog Commun Health Partnerships Res Educ Act. 2007;1 (4):371-81.

33. Pérez-Escamilla R, Hromi-Fiedler A, Vega-López S, Bermúdez-Millán A, Segura-Pérez S. Impact of peer nutrition education on dietary behaviors and health outcomes among Latinos: a systematic literature review. J Nutr Educ Behav. 2008;40(4):208-25.

34. Wells KJ, Luque JS, Miladinovic B, et al. Do community health worker interventions improve rates of screening mammography in the United States? A systematic review. Cancer Epidemiol Prev Biomark. 2011;20(8):1580-98.

35. Office of Disease Prevention and Health Promotion. Eat Healthy, Be Active Community Workshops. 2016.

36. U.S. Census. QuickFacts: Yakima County, Washington. 2017.

37. Thompson B, Coronado GD, Solomon CC, McClerran DF, Neuhouser ML, Feng Z. Cancer prevention behaviors and socioeconomic status among Hispanics and non-Hispanic whites in a rural population in the United States. Cancer Causes Control. 2002;13(8):719-28. 
38. Viechtbauer W, Smits L, Kotz D, et al. A simple formula for the calculation of sample size in pilot studies. J Clin Epidemiol. 2015;68(11):1375-9.

39. Duggan C, Carosso E, Mariscal N, et al. Diabetes prevention in Hispanics: report from a randomized controlled trial. Prev Chronic Dis. 2014;11(11):E28

40. Thompson FE, Subar AF, Smith AF, et al. Fruit and vegetable assessment: performance of 2 new short instruments and a food frequency questionnaire. J Am Diet Assoc. 2002;102(12):1764-72.

41. Centers for Disease Control and Prevention. Healthy weight, nutrition, and physical activity. 2020.

42. Harris PA, Taylor R, Thielke R, Payne J, Gonzalez N, Conde JG. Research electronic data capture (REDCap)-a metadata-driven methodology and workflow process for providing translational research informatics support. J Biomed Inform. 2009;42(2):377-81.

43. Cha E, Kim KH, Lerner HM, et al. Health literacy, self-efficacy, food label use, and diet in young adults. Am J Health Behav. 2014;38(3):331-9.

44. Sharif MZ, Rizzo S, Prelip ML, et al. The association between nutrition facts label utilization and comprehension among Latinos in two east Los Angeles neighborhoods. J Acad Nutr Diet. 2014;114(12):1915-22.

45. Campos S, Doxey J, Hammond D. Nutrition labels on pre-packaged foods: a systematic review. Public Health Nutr. 2011;14(8):1496-506.

46. Persoskie A, Hennessy E, Nelson WL. US consumers' understanding of nutrition labels in 2013: the importance of health literacy. Prev Chronic Dis. 2017:14:E86.

47. Malik VS, Popkin BM, Bray GA, Després JP, Willett WC, Hu FB. Sugar-sweetened beverages and risk of metabolic syndrome and type 2 diabetes: a meta-analysis. Diabetes Care. 2010;33(11):2477-83.
48. Chen L, Caballero B, Mitchell DC, et al. Reducing consumption of sugar-sweetened beverages is associated with reduced blood pressure: a prospective study among United States adults. Circulation. 2010;121(22):2398-406.

49. Dauchet L, Amouyel P, Hercberg S, Dallongeville J. Fruit and vegetable consumption and risk of coronary heart disease: a meta-analysis of cohort studies. J Nutr. 2006;136(10):2588-93.

50. Bautista L, Reininger B, Gay JL, Barroso CS, McCormick JB. Perceived barriers to exercise in Hispanic adults by level of activity. J Phys Act Health. 2011;8(7):916-25

51. Foster-Schubert KE, Alfano CM, Duggan CR, et al. Effect of diet and exercise, alone or combined, on weight and body composition in overweight-to-obese postmenopausal women. Obesity (Silver Spring). 2012;20(8):1628-38.

52. Robert Wood Johnson Foundation. The State of Obesity 2016: Better Policies for a Healthier America. 2016.

53. Sylvia LG, Bernstein EE, Hubbard JL, Keating L, Anderson EJ. Practical guide to measuring physical activity. J Acad Nutr Diet. 2014;114(2):199-208.

54. Thompson FE, Subar AF. Nutrition in the prevention and treatment of disease. London: Academic Press; 2017.

\section{Publisher's Note}

Springer Nature remains neutral with regard to jurisdictional claims in published maps and institutional affiliations.
Ready to submit your research? Choose BMC and benefit from:

- fast, convenient online submission

- thorough peer review by experienced researchers in your field

- rapid publication on acceptance

- support for research data, including large and complex data types

- gold Open Access which fosters wider collaboration and increased citations

- maximum visibility for your research: over 100M website views per year

At BMC, research is always in progress.

Learn more biomedcentral.com/submissions 